\title{
Fluctuations, Correlations and Non-Extensivity
}

\author{
Grzegorz Wilk \\ The Andrzej Soltan Institute for Nuclear Studies, Hoża 69, 00681 Warsaw, Poland
}

Received on 23 October, 2006

\begin{abstract}
The present status of investigations on fluctuations and correlations seen in high energy multiparticle production processes made using the notion of nonextensivity is reviewed.
\end{abstract}

Keywords: Fluctuations; Correlations; Nonextensivity

\section{INTRODUCTION}

There are many examples that distributions of produced particles deviate from the expected exponential form towards its power-like generalization [1], called the $q$-exponential and know also as Tsallis distribution [2],

$$
\exp _{q}\left(-\frac{X}{\lambda}\right) \stackrel{\text { def }}{=}\left[1-(1-q) \frac{X}{\lambda}\right]^{1 /(1-q)} \stackrel{q \rightarrow 1}{\Longrightarrow} \exp \left(-\frac{X}{\lambda}\right)
$$

described by parameter $q$ (in cases of interest to us $X=p_{T}$ or $X=m_{T} \cosh y$ ). Distributions of this type were proposed on phenomenological grounds long time ago as useful parametrization of data interpolating between results coming from soft and hard scattering [3]. On the other hand, when seen from the information-theory point of view or from the thermodynamical approach, such distributions arise from the use of non-extensive Tsallis entropy [4] (indexed by $q$ ) instead of the usual Shannon-Gibbs-Boltzmann one:

$$
S_{q}=-\frac{1}{1-q}\left(1-\sum_{i} p_{i}^{q}\right) \stackrel{q \rightarrow 1}{\Longrightarrow} S_{S B G}=-\sum_{i} p_{i} \ln p_{i} .
$$

Notice that $S_{q}$ is nonextensive in such sense that for two independent systems, $A$ and $B$ (in the usual sense, i.e., that the corresponding probabilities of their occurrence factorize, $\left.p_{i j}(A+B)=p_{i}(A) p_{j}(B)\right)$ one has that

$$
S_{q}(A+B)=S_{q}(A)+S_{q}(B)+(1-q) S_{q}(A) S_{q}(B) .
$$

The entropic index $q$ is thus also a measure of nonextensivity in system under consideration and therefore is also called nonextensivity parameter.

What are the circumstances leading to $q \neq 1$ ? The common consensus is that it happens whenever [4]: - there are long range correlations in the system (or system is small - notice that our Universe is small with respect to the gravitational interactions!); - there are memory effects of different kind; - the phase-space in which system operates is limited or has fractal structure and, finally, - there are intrinsic fluctuations in the system under consideration.

\section{THE ROLE OF INTRINSIC FLUCTUATIONS}

Our works, which I shall review now, were concerned with the last possibility mentioned above, namely that $|1-q|$ measures fluctuations in the hadronizing system under consideration. As shown in [5] in the case when these fluctuations can be described by gamma distribution,

$$
\begin{aligned}
& {\left[1-(1-q) \frac{\varepsilon}{\lambda}\right]^{1 /((1-q)}=\int_{0}^{\infty} e^{-\frac{\varepsilon}{\lambda}} f(\beta) d \beta} \\
& f(\beta)=\frac{1}{\Gamma(\alpha)}\left(\frac{\alpha}{\langle\beta\rangle}\right)^{\alpha} \beta^{\alpha-1} e^{-\frac{\alpha}{\langle\beta\rangle} \beta}, \quad \alpha=\frac{1}{q-1} \\
& q=1 \pm \frac{\left\langle\beta^{2}\right\rangle-\langle\beta\rangle^{2}}{\langle\beta\rangle^{2}}
\end{aligned}
$$

where $\langle\ldots\rangle$ are the respective averages taken with respect to $f$. In general case one refers to the concept of the so called superstatistics discussed at length in [6]. This approach has been recently further generalized in [7].

The situation encountered can be visualized in the following way. Already in [8] it was suggested that the overwhelmingly success of thermodynamical models has its origin in the fact that out of the large number $N$ of secondaries produced in a given event only one (very rarely two) is chosen for making distributions. Inevitably, the remain $(N-1)$ act as a kind of heat bath which action can in most cases be described by single parameter $T$ (called usually temperature because of associations with thermal models). In this case one has simple exponential distributions (i.e., $q=1$ ). However, because such "heat bath" is in reality neither homogeneous nor infinite, it is natural to expect that it should be described by more parameters. The simplest extension is to assume that $T$ fluctuates, replace $T \rightarrow T_{0}=\langle T\rangle$ and introduce another (second) parameter describing its fluctuations - this leads to $q \neq 1$ in most natural way. Mathematically it can be realized by introducing to the well-known linear Langevin equation with additive noise, modelling the Brownian motion of some test particle (and leading to a Boltzmann distribution as stationary solution of the corresponding Fokker-Planck (FP) equation), a small multiplicative noise. This leads immediately to Tsallis distributions of relevant observable as exact stationary solution of the corresponding FP equation with $(q-1)$ being directly connected with the strength of this new term (cf. [5, 7]). To close this point it must be mentioned that the role of correlations is so far not excluded but it seems that in the realm of high energy multiparticle production they are, at least at circumstances considered so far, not the dominant component $[9,10]$.

Experimental data of interest come usually in two categories: as distributions in longitudinal phase space (usually in rapidity $y$ ) averaged over transverse momenta, $d N / d y$, or distributions in transverse momenta, $d N / d p_{T}$, averaged over $y$ or taken for some window in $y$. We have therefore also two kinds 
of "temperatures", $T_{L}$ and $T_{T}$, and their fluctuations are thus described by, respectively, $q_{L}$ and $q_{T}$. Out of these two classes only exponential shape of $d N / d p_{T}$ spectra is usually regarded as indication of thermal-like character of such processes with a kind of local thermal equilibrium setting in and characterized by temperature $T=T_{T}$, which can be deduced from the corresponding slopes of $d N / d p_{T}$. Any deviation from them is then interpreted as signal for some dynamical (nonequilibrium) effects showing up (like, for example, the flow or decay of resonances, see [11] and references therein). Instead of trying to exclude them one can investigate the possibility that the observed nonexponential spectra result from some new form of equilibrium characteristic of nonextensive thermodynamics (incorporating, among others, also effects of the aforementioned factors). The plausibility of such approach is illustrated
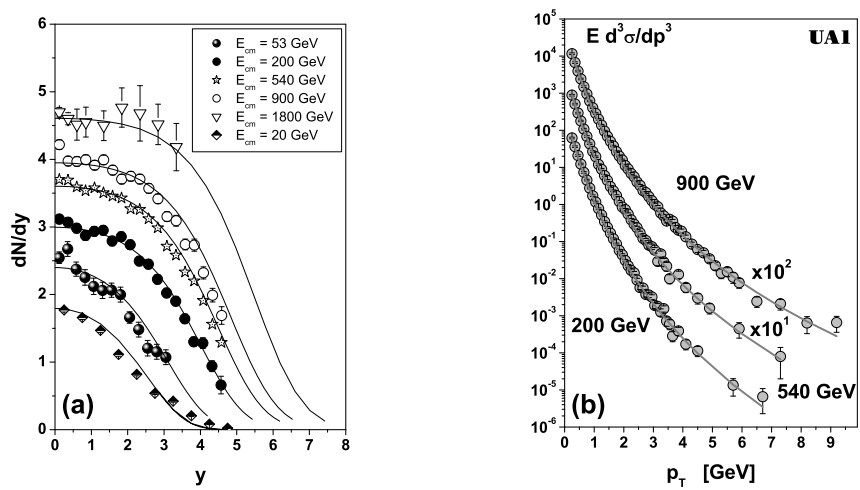

FIG. 1: Examples of Tsallis distributions: $(a)$ fit to rapidity spectra for charged pions produced in $p p$ and $\bar{p} p$ collisions at different energies. (b) fit to $p_{T}$ spectra from UA1 experiment (see [12] for details and references to experimental data).

in Fig. 1 where examples of both types of distributions are shown [12]. The characteristic feature encountered here is that $q_{L}>q_{T}$ and that $T_{L}>>T_{T}$ ( $q_{L}$ ranges in linear fashion from 1.05 for $\sqrt{s}=20 \mathrm{GeV}$ to 1.33 for $\sqrt{s}=1800 \mathrm{GeV}$ whereas variation of $q_{T}$ is limited to between 1.095 for $\sqrt{s}=200 \mathrm{GeV}$ and 1.11 for $\sqrt{s}=900 \mathrm{GeV}$; the respective changes of $T_{L}$ are from $1.76 \mathrm{GeV}$ to $55.69 \mathrm{GeV}$ whereas those of $T_{T}$ are much smaller varying from $0.134 \mathrm{GeV}$ to $0.14 \mathrm{GeV}$ ). The immediate question arises: what is the meaning of $q_{L}$, or, equivalently, what is the meaning of fluctuations of "partition temperature" $T_{L}$ ? The answer is that $q_{L}=1+1 / k$, where $k$ is parameter defining (in addition to mean multiplicity $\langle n\rangle$ ) the Negative Binomial distributions of multiplicity observed in such collisions [16]. It is because $T_{L} \sim M /\langle n\rangle$, where $M$ is energy available for production of secondaries, therefore for $M$ kept constant fluctuations of $T_{L}$ mean automatically fluctuations of $\langle n\rangle$ and those lead immediately to NBD [16]. Interestingly enough NBD arises also in a natural way when particles are produced with energies distributed according to Tsallis distribution (when they are distributed according to Boltzmann distributions one gets Poisson distribution instead, see [9]).

Let us proceed now to recent RHIC data on $p_{T}$ distributions, which consist our potential source of the parameter $T$, as mentioned above. As shown in [13], using approach based either on nonextensive statistics or on stochastic ideas one can successfully account for the whole range of the observed transverse momenta. This is because in both cases the resultant distributions are intrinsically non-exponential. In [14] the same data were analyzed using Hagedorn model with spectrum of resonances given by $\rho(m)$ :

$$
\begin{aligned}
\frac{d^{3} \sigma}{d p^{3}} & =C \int d m \rho(m) \exp \left(-\beta_{0} \cdot \sqrt{p_{l}^{2}+p_{t}^{2}+m^{2}}\right) \\
\rho(m) & =\frac{\exp \left(m \beta_{H}\right)}{\left(m^{2}+m_{0}^{2}\right)^{5 / 4}}
\end{aligned}
$$

As seen in Fig. 2, although $\rho(m)$ introduces already some
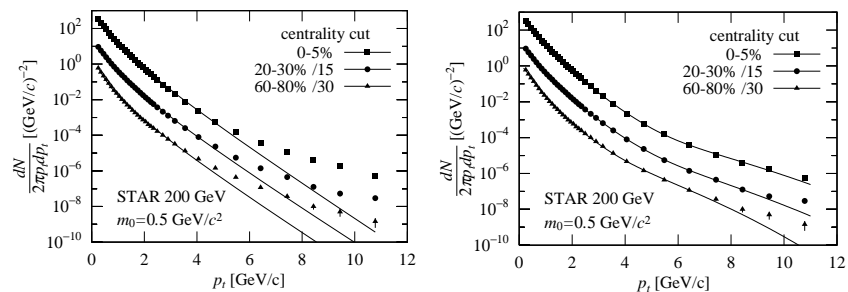

FIG. 2: Example of analysis of STAR data [15] by using usual Hagedorn formula (eq. (7) with $q=1$, left panel) and its nonextensive generalization (eq. (7) with $q>1$, right panel), see [14] for details.

fluctuations to the system, experimental data can be fitted only with $q_{T}>1$ (of the order of $q_{T} \simeq 1.00015$ ). The new and potentially very interesting fact is that similarly good fit can be obtained with simple $q$-exponential (i.e., by putting $\rho(m)=1$ in eq. (7)), however, in this case $q$ is noticeably greater and equal to $q_{T}=1.065$. This effect is seen for all RHIC data and for all centralities. It strongly suggests that including some well identified sources of fluctuations to simple statistical model (represented here by function $\rho(m)$ as given by eq. (8)) accounts for some fluctuations and lowers therefore the value of parameter $q_{T}$. However, as seen in Fig. 2, data are very sensitive to $q$ and, as has been shown in [14], to obtain good estimation of $T_{0}=1 / \beta_{0}$ one has to resort to nonextensive version of eq. (7) with $q_{T}>1$.

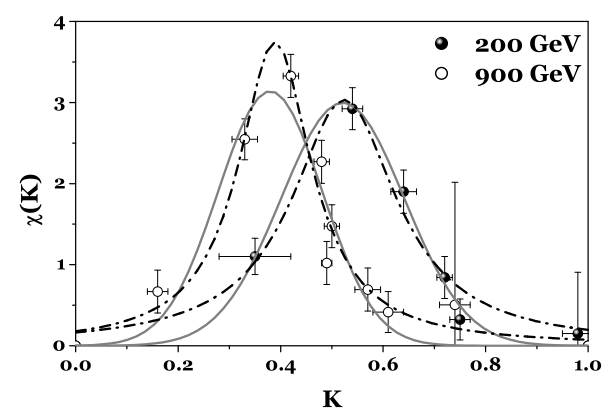

FIG. 3: Example of EGE [17]: Inelasticity distributions $\chi(K)$ (normalized to unity) obtained in [16] from analysis of multiparticle production data for $\sqrt{s}=200 \mathrm{GeV}$ and $\sqrt{s}=900 \mathrm{GeV}$ fitted by gaussian (full lines) and lorentzian (dashed lines) distributions, respectively (see [16] for more details).

We would like to close this Section by mentioning that there is yet another source of fluctuation in the production process, 
which also leads to nonextensivity but not of Tsallis type, i.e., with gaussian distributions instead of eq. (1). Their source lies in the fact that usually only fraction $K$ (called inelasticity) of the total energy of reaction is used for production of secondaries and this fraction can fluctuate. It turns out that such fluctuations are most naturally described in the frame of the so called Extended Gaussian Ensemble (EGE) by simple gaussian distribution, $\chi(K) \simeq \exp \left[-(K-\langle K\rangle)^{2} /\left(2 \sigma^{2}\right)\right]$, (cf. [17] for details and references) and that they can reasonably fit distributions of $\chi(K)$ for different energies obtained in [16], see Fig. 3. In fact, closer inspection of Fig. 3 shows that still better fit can be obtained when using the so called lorentzian distribution, which is nothing else as $q$-gaussian with $q=2$. It would mean then that even here there are still some other fluctuations seen in data, which are undisclosed by the usual EGE approach.

\section{SUMMARY}

To summarize: we argue (since quite a time already) that whenever one finds in data a hint for eq. (1) the immediate suspicion should be that they hide some intrinsic fluctuations with strength given by $q-1$. What is the dynamical origin of these fluctuations is, however, out of the scope of the procedure discussed here as $q$ accounts for all of them. The $q>1$ results should then urge experimentalists to devise some special measurements devoted to search for fluctuations.

To allow the reader to make personal judgement in what concerns the possible role of correlations let us list recent at- tempts to interpret parameter $q$ by some dynamical correlations caused either by incomplete phase space occupance [18] or by some specific changes introduced in generalized form of the Boltzmann equation (either by using corresponding collision rates nonlinear in the one-particle densities or by using nontrivial energy composition rules in the energy conservation constraint part, cf. [19] and references therein). It should also be added at this point that, as advocated in [20], one can also view $q>1$ as a general (leading order) finite-size effect.

Finally, one should be aware of the fact that there is still an ongoing discussion on the meaning of the temperature in nonextensive systems. However, the small values of parameter $q_{T}$ deduced from data allow us to argue that, to first approximation, $T_{0}$ can be regarded as the hadronizing temperature in such system. One must only remember that in general what we study here is not so much the state of equilibrium but rather some kind of stationary state (see [21] and references therein).

Let us close by saying that this subject is still an open issue for further research (like, for example, event-by-event analysis of data [22] or hydrodynamical models [23]).

\section{Acknowledgements}

The subject reviewed here has been investigated in collaboration with O.Utyuzh, Z.Włodarczyk, F.S.Navarra, M.Biyajima, M.Kaneyama, T.Mizoguchi, N.Nakamija, N.Suzuki and T.Osada. Partial support (GW) of the Ministry of Science and Higher Education (grants Nr 621/E78/SPB/CERN/P-03/DWM 52/2004-2006 and 1 P03B 022 30) is acknowledged.
[1] I. Bediaga, E. M. Curado, and J. M. de Miranda, Physica A 286, 156 (2000); W. M. Alberico, A. Lavagno, and P. Quarati, Eur. Phys. J. C 12, 499 (2000); G. Wilk and Z. Włodarczyk, Nucl. Phys. B (Proc. Suppl.) A 75, 191 (1999); Physica A 305, 227 (2002); F. S. Navarra, O. V. Utyuzh, G. Wilk, and Z. Włodarczyk, Nuovo Cim. C 24, 725 (2001); T. Wibig and I. Kurp, Int. J. High Energy Phys. 0312, 039 (2003).

[2] C. Tsallis, Braz. J. Phys. 29, 1 (1999).

[3] C. Michael and L. Vanryckeghem, J. Phys. G 3, L151 (1977); C. Michael, Prog. Part. Nucl. Phys. 2, 1 (1979); G. Arnison et al. [UA1 Collaboration], Phys. Lett. B 118, 167 (1982); R. Hagedorn, Riv. Nuovo Cim. 6 (10), 1 (1983); P. Steinberg et al., Expression of interest for a comprehensive new detector at RHIC II, arXiv:nucl-ex/0503002.

[4] Cf., for example, C. Tsallis, Physica A 340, 1 (2004), A 344, 718 (2004) and A 365, 7 (2006) and references therein. See also special issue of Europhysics News, Nov-Dec. 2005 (EPSP) (http://www.europhysicsnews.com).

[5] G. Wilk and Z. Włodarczyk, Phys. Rev. Lett. 84, 2770 (2000) and Chaos, Solitons and Fractals 13/3, 581 (2001).

[6] C. Beck and E. G. D. Cohen, Physica A 322, 267 (2003); F. Sattin, Eur. Phys. J. B 49, 219 (2006).

[7] T. S. Biro and A. Jakovác, Phys. Rev. Lett. 94, 132302 (2005).

[8] L. Van Hove, Z. Phys. C 21, 93 (1985) and C 27, 135 (1985).

[9] G. Wilk and Z. Włodarczyk, Physica A 376, 279 (2007).

[10] K. E. Bassler, G. H. Gunarante, and J. L. McCauley, MarkoveProcesses, Hurst Exponents, and Nonlinear Diffusion Equa- tions with application to finance, cond-mat/0602316.

[11] B. Müller and J. L. Nagle, Ann. Rev. Nuc. Part. Sci. 56, 93 (2006).

[12] F. S. Navarra, O. V. Utyuzh, G. Wilk, and Z. Włodarczyk, Physica A 340, 467 (2004) and A 344, 568 (2004).

[13] M. Biyajima, M. Kaneyama, T. Mizoguchi, and G. Wilk, Eur. Phys. J. C 40, 243 (2005)

[14] M. Biyajima, T. Mizoguchi, N. Nakajima, N. Suzuki, and G. Wilk, Eur. Phys. J. C 48, 597 (2006).

[15] J. Adams et al. [STAR Collaboration], Phys. Rev. Lett. 91, 172302 (2003).

[16] F.S. Navarra, O.V. Utyuzh, G. Wilk, and Z. Włodarczyk, Phys. Rev. D 67, 114002 (2003).

[17] T. Osada, O.V. Utyuzh, G. Wilk and Z. Włodarczyk, Eur. Phys. J. B 50, 7 (2006).

[18] T. Kodama, H.-T. Elze, C.E. Augiar and T. Koide, Europ. Lett. 70, 439 (2005); V. Garcia-Morales and J. Pellicer, Physica A 361, 161 (2006).

[19] T. S. Biro and G. Kaniadakis, Eur. Phys. J. B 50, 3 (2006).

[20] A. S. Parvan, Phys. Lett. A 350, 331 (2006); Phys. Lett. A 360, 26 (2006).

[21] S. Abe, Physica A 368, 430 (2006).

[22] O. V. Utyuzh, G. Wilk, and Z. Włodarczyk, J. Phys. G 26, L39 (2000).

[23] T. Osada and G. Wilk, in preparation. 